\title{
Antihormonal agents as a strategy to improve the effect of chemo-radiation in cervical cancer: in vitro and in vivo study
}

Mariana Segovia-Mendoza ${ }^{1,2}$, Rafael Jurado ${ }^{1}$, Roser Mir ${ }^{1,3}$, Luis A Medina ${ }^{3,4}$, Heriberto Prado-Garcia $^{5}$ and Patricia Garcia-Lopez ${ }^{1 *}$

\begin{abstract}
Background: Over the past few years, the concurrent use of cisplatin-based chemotherapy and radiation therapy has dramatically improved the local response and increased overall survival in early-stage cervical cancer. However, for the advanced stages of the disease this standard treatment has proved insufficient. We investigated the capacity of Mifepristone and ICI 182,780, which are anti-progestin and anti-estrogen drugs, respectively, to act as chemoradiosensitizing agents in cervical cancer cells and cervix xenografts.

Methods: The effect of chemo-radiation alone or combined with Mifepristone or ICI 182,780 was evaluated in HeLa cells and with tumor growth in cervix xenografts. After concomitant chemo-radiotherapy, the effect of each of these antihormonal agents on apoptosis (determined by Annexing $\vee$ assay) and the cell cycle phases were determined by flow cytometry. The expression of angiogenic factor VEGF in tumor samples was determined using quantitative RT-PCR analysis of VEGF gene expression.

Results: Compared to radiation alone or radiation/cisplatin therapy, there was significantly higher cytotoxicity and a greater antitumoral effect with the combined application of radiation/cisplatin and Mifepristone or ICI 182,780. Analyses of the apoptosis and cell cycle demonstrated changes only with $\mathrm{ICl}$, not with Mifepristone, when was applied in combination with radiation/cisplatin. The analysis of VEGF mRNA expression levels in tumors at the end of the study demonstrated a significant inhibition, compared to radiation only or the radiation/cisplatin treatment, after concurrent chemo-radiotherapy and each one of the antihormonal drugs.
\end{abstract}

Conclusion: Mifepristone and ICI 182,780 may be potentially promising chemo-radiosensitizing compounds to be used in combination with ionizing irradiation and cisplatin in the treatment of patients with advanced cervical cancer.

Keywords: Cervical cancer, Mifepristone, ICI 182,780, Cisplatin, Radiosensitizing

\section{Background}

Cervical cancer is still a major cause of morbidity and mortality in women worldwide. In several low-income countries this disease, if not treated in time, is one of the most aggressive gynecological cancers. Although routine screening programs have been implemented since 1975, there continues to be an increased rate of new cases [1,2]. Human papillomavirus (HPV) has been proposed as an etiological factor in the pathogenesis of this cancer [3].

\footnotetext{
* Correspondence: pgarcia_lopez@yahoo.com.mx

${ }^{1}$ Instituto Nacional de Cancerología, Subdirección de Investigación Básica, México D.F. 14080, México

Full list of author information is available at the end of the article
}

At present, cervical cancer is considered as a potentially curable disease in cases of early detection. Unfortunately the majority of cases are diagnosed in the metastatic or advanced stage, implying a worse prognosis that requires a systemic treatment. Currently, the treatment for cervical carcinoma is the combination of cisplatin-based chemotherapy and radiotherapy $[4,5]$. The introduction of chemo-radiation for early stage cervical carcinoma led to improvements in survival. Different studies have demonstrated that chemo-radiotherapy lead to a significant survival advantage of $10-15 \%$ at 5 years post-treatment compared with radiotherapy alone for patients in stage 
IB-IIB [6]. However, the prognosis for patients in stage IIIA-IVA is still unfavorable [6-8].

Apart from their limited effectiveness in the advanced stage of the disease, current chemotherapy and radiotherapy have serious drawbacks. The administration of cisplatin is associated with serious side effects, such as nephrotoxic and neurotoxic events [9], and the effectiveness of radiation therapy is limited by damage to the normal tissue. Several studies have sought to find drugs capable of potentiating the antiproliferative effect of chemo-radiotherapy in cervical cancer. In spite of the promising anti-tumoral results with the use of hydroxyurea, gemcitabine or taxanes as radiosensitizers, even greater side effects have been found with these drugs. Hence, ongoing efforts are necessary to further improve the outcome with locally advanced cervical cancer by maximizing the local response and patient survival.

One proposal of this work is to seek chemo- and radio- sensitizer drugs with less adverse effects. Some antihormonals, such as tamoxifen, raloxifene, medroxiprogesterone, mifepristone and ICI 182,780, have been used in the treatment of hormone-dependent cancers (e.g., breast, ovarian, prostate and endometrial). However, these compounds have been poorly studied as chemoradiosensitizers in cervical cancer because this carcinoma is traditionally considered not to respond to antihormonal therapy [10]. Nevertheless, the mechanisms of action of these antihormonal agents described in the literature seem to hold promise in facilitating the response to chemo-radiation in cervical cancer cells. Such mechanisms include caspase activation, growth factor regulation [11], anti-apoptotic proteins inhibition [12,13] and apoptosis induction by $\mathrm{p} 53[14,15]$.

The possible role of mifepristone (MF) or ICI 182,780 (ICI) to enhance the cytotoxicity of cisplatin and the effectiveness of radiotherapy in cervical cancer treatment has not been sufficiently explored. MF has activity on progesterone and is a glucocorticoid receptor antagonist. It has been used a chemosensitizing drug to modulate the cytotoxic activity of doxorubicin [16], paclitaxel [17] and cisplatin on ovarian cancer cells [18]. We previously demonstrated that MF was able to enhance the cytotoxicity of cisplatin in cervical cancer cells in-vitro and invivo by increasing the intracellular and intratumoral concentration of cisplatin [19]. Furthermore, we recently reported a reduction in the rate of tumor growth when MF was added to the temozolamide-radiation scheme in glioblastoma xenografts [20]. Overall, this evidence suggests that MF could play an important role as chemoand radiosensitizer.

In another study using cervical cancer cells [21], we showed that the combination of cisplatin with the antiestrogen ICI induced the arrest of the cell cycle at the G2/M phase. The failure of this control checkpoint may lead to genomic instability resulting in hypersensitivity to radiation.

The aim of the present study was to evaluate whether MF or ICI used simultaneously with cisplatin and radiotherapy could show a chemo-radiosensitizer effect, increasing the anti-proliferative effect in cervical cancer cells and in xenostransplants when treated with cisplatin and radiation. To correlate the mechanism of action of these antihormonals in the modulation of the effects of chemo-radiotherapy on tumor cells, an analysis of cell cycle and apoptosis at different times was made (the latter evaluated by Annexin V binding assay). Additionally, the growth of cervix xenotrasplants was correlated with a decrease in VEGF gene expression.

\section{Methods}

\section{Drugs and reagents}

Cisplatin, Mifepristone, Chloroform, Trypsin and Sodium Chloride were obtained from Sigma Chemical Co. (St. Louis, MO, USA). ICI 182,780 was obtained from Tocris Cookson, Inc. (Balwin, MO, USA). Dulbecco's modified Eagle's medium (DMEM), FCS (fetal calf serum), EDTA (Ethylenediaminetetracetic acid), Tris and SDS were obtained from Gibco, BRL (Grand Island, NY, USA). High-quality water employed to prepare solutions was obtained through of a Milli-Q Reagent Water System Continental Water Systems (El Paso, TX, USA). Taq DNA polymerase was purchased from Invitrogen (Carlsbad, CA, USA).

\section{Solutions}

A stock solution $(1 \mathrm{mg} / \mathrm{mL})$ of cisplatin was prepared in saline solution. ICI and MF were reconstituted in absolute ethanol (stock solution). All standard solutions were stored at $-20^{\circ} \mathrm{C}$ until use.

\section{Animals}

Female athymic Balb-c nu/nu mice, between 6-8 weeks of age, were supplied by the Instituto Nacional de Nutrición (INCMNSZ), Mexico City, Mexico. All animals were kept in a pathogen-free environment and fed $a d l i b$. The procedures for care and use of animals were approved by the Ethics Committee of the Instituto Nacional de Cancerología (INCan, Mexico City, Mexico) and all applicable institutional and governmental regulations concerning the ethical use of animals were followed.

\section{Cell cultures}

The HeLa human cervical cancer cell line was obtained from ATCC (Rockville, Maryland, USA), and was routinely maintained as a monolayer in DMEM supplemented with $10 \%$ fetal bovine serum, and incubated at $37^{\circ} \mathrm{C}$ with high humidity in a $5 \% \mathrm{CO}_{2}$ atmosphere. Cells were harvested with $0.025 \%$ trypsin and $1 \mathrm{mM}$ EDTA. 


\section{Chemo-radiotherapy on HeLa cells}

Cells were seeded into $25 \mathrm{~cm}^{2}$ culture plates at a density of $5 \times 10^{5}$ cells per well and pretreated for 3 days with $10 \mu M$ MF or ICI. Control cells were exposed only to the vehicle (the final ethanol concentration never exceeded $1 \% \mathrm{v} / \mathrm{v}$ in treated or control samples). At the end of the exposure period, the culture medium was removed and fresh medium with $0.33 \mu M$ of cisplatin plus MF or ICI was added. After $24 \mathrm{~h}$ the cells were irradiated at 0.75 Gy using a ${ }^{60}$ Co irradiator (Theraton, Phoenix, USA). Controls were handled in the same way as the irradiated cultures. Moreover, cells exposed only to the individual treatments (with or without radiation) served as a positive control. After exposure, cell survival was determined by the clonogenic assay.

\section{Clonogenic cell survival assay}

Surviving cells were analyzed for cytotoxic effects of chemotherapy and/or irradiation treatments, according to the establish method of the clonogenic assay [22]. Briefly, following exposure to radiation, the cells were immediately rinsed, trypsinized, diluted, counted, and seeded in triplicate at different cell densities in $25 \mathrm{~cm}^{2}$ plates, and then allowed to grow at $37^{\circ} \mathrm{C}$ for 2 weeks. During this time colonies were formed from surviving cells, and these colonies were fixed in $10 \%$ formaldehyde and stained with a solution of crystal violet. Colonies of 50 cells or more were counted manually with a cell counter (Bantex, USA). The survival rate was expressed as the plating efficiency (PE), which is equal to the average number colonies counted divided by the total number of cells plated a calculation that normalized clonogenic survival. At least three independent experiments were performed for each assay.

\section{Cell cycle analysis}

The cells $\left(5 \times 10^{4}\right)$ were synchronized and plated in specific medium following the previously described protocol. At 24, 48 and $72 \mathrm{~h}$ post-irradiation, the cultured cells were harvested and washed twice with PBS, then fixed with $70 \%(\mathrm{v} / \mathrm{v})$ ethanol and stored at $4^{\circ} \mathrm{C}$ overnight. Afterwards, ethanol was removed and the samples were washed with PBS. Cellular DNA staining was performed with Guava Cell Cycle Reagent for 30 min (Guava Technologies, Millipore, Hayward, CA, USA). The data were collected and analysed by a Guava EasyCyte Flow cytometer with use of GuavaSoft software (Millipore, Hayward, CA), as a minimum $1 \times 10^{4}$ cells were acquired. At least three independent experiments were performed.

\section{Annexin V staining assay for apoptosis}

Phosphatidylserine externalization was analyzed using the Guava Nexin Kit. Cells were plated and treated as before mentioned, the Annexin $\mathrm{V}$ binding assay was conducted at 24, 48 and $72 \mathrm{~h}$ post-irradiation. Cultured cells were harvested and washed twice with PBS, and then resuspended in Guava Nexin Reagent, containing binding buffer, Annexin V-phycoerytrin (PE) and 7aminoactinomycin D (7-AAD), incubated for $20 \mathrm{~min}$ at room temperature according to Guava protocol instructions (Guava Technologies, Millipore, Hayward, CA, USA). Analysis was carried out by flow cytometry using Guava EasyCyte, acquiring $5 \times 10^{3}$ cells. Annexin analysis was performed with the GuavaSoft software (Millipore, Hayward, CA). At least three independent experiments were performed for each assay.

\section{Tumor xenografts}

Mice were subcutaneously (s.c) inoculated with $5 \times 10^{6}$ HeLa cells in both hind limbs. The limbs were selected as the site for tumor growth to minimize irradiation to organs. Weekly measurements of tumors were made after inoculation. Two perpendicular diameters were measured by using a caliper, and tumor volume was determined by using the following relation: $\mathrm{V}=\pi / 6 \times$ (large diameter $\left.\times[\text { short diameter }]^{2}\right)$. Once tumors had reached approximately $150 \mathrm{~mm}^{3}$, the animals were pair-matched in treatment and control groups and the treatments were initiated. Each group consisted of 5-6 tumor-bearing mice.

\section{Irradiation procedure}

Animals were anaesthetized with $1-3 \%$ isoflurane in $100 \%$ oxygen by using an animal anesthesia inhalation unit (Bickford, Wales Center, NY, USA), and irradiated with an orthovoltage X-ray unit (D3225, Gulmay Medical Ltd., UK) as described previously [23]. Animals received fractionated doses of 0.5 Gy per day for 20 days (Monday through Friday for four weeks). The dose and schedule were selected in accordance with the doseresponse curve constructed in a previous pilot study. This curve showed a 10 Gy dose as the $\mathrm{ED}_{50}$ (dose of radiation to achieve $50 \%$ growth inhibition). The X-ray beam was centered on the tumor lobe by using one of the different lead collimators [23], depending on the tumor size at the moment of irradiation.

\section{Chemo-radiotherapy in tumor xenografts}

Animals selected for this study were divided into eight groups ( $\mathrm{n}=5-6$ each) including: A) radiation treatment alone (0.5 Gy/day for 20 days); B) cisplatin treatment alone (3 $\mathrm{mg} / \mathrm{kg} /$ week for three cycles, i.p.); C) MF alone ( $2 \mathrm{mg} / \mathrm{kg} /$ day, s.c.) or ICI alone (100 mg/kg/day, s.c.); D) irradiation combined with cisplatin; E) irradiation combined with MF or ICI; F) cisplatin combined with MF or ICI; and G) three treatments combined (cisplatin/radiation/ICI or cisplatin/radiation/MF). ICI and MF were administered in three cycles during three weeks; each 
cycle consisted of three consecutive days (Monday through Wednesday). Control animals received only the vehicle and no irradiation. Mice were weighed and the tumor volume was calculated every five days as previously described. The experiment was conducted during 10 weeks, at the end of which time all animals were weighed and euthanized.

\section{VEGF expression analysis by quantitative reverse transcription-PCR (qRT-PCR)}

The effect of MF or ICI on the expression of angiogenic factors during concomitant chemo-radiotherapy was examined using qRT-PCR. VEGF expression levels in tumor tissue from cervical carcinoma xenografs were evaluated at the end of the study. Briefly, whole tumors were lysed and the total RNA was isolated from each tumor with a method based on guanidine isothiocyanate/ phenol/chloroform extraction using the TRIzol reagent (Invitrogen Life Technologies), and then quantified with UV spectroscopy. After quantification, $200 \mathrm{ng}$ of the total RNA was used in presence of the TaqMan ${ }^{\circ}$ RNA-to-CT ${ }^{\mathrm{mm}}$ 1-Step Kit (Applied Biosystems) to perform one-step RTPCR TaqMan Gene Expression Assays of vascular endothelial growth factor A (VEGF-A) (Hs00900055_ml, Applied Biosystems) by using a FAM probe and Endogenous Control Human GAPDH (4310884E, Applied Biosystems) with VIC. Real-time quantification was performed on a Spectrum 48 thermocycler Instrument (ESCO, Micro Pte Ltd, Singapore).

PCR reactions were carried out in a total volume of $10 \mu \mathrm{l}$. The reaction conditions were as follows: preincubation at $60^{\circ} \mathrm{C}$ for 15 minutes and $94^{\circ} \mathrm{C}$ for $5 \mathrm{mi}$ nutes, followed by 40 cycles (amplification) of $94^{\circ} \mathrm{C}$ for $15 \mathrm{~s}$ and $60^{\circ} \mathrm{C}$ for $60 \mathrm{~s}$. Fluorescence emission spectra were monitored and analyzed. PCR products were measured at the threshold cycle $(\mathrm{Ct})$, at which time specific fluorescence became detectable. The $\mathrm{Ct}$ was used for kinetic analysis and was proportional to the initial number of target copies in the sample. Analysis of relative gene expression was based on the $2^{-\Delta \Delta C t}$ method and was carried out with three independent samples.

\section{Statistical analysis}

Values are reported as the mean \pm SEM (standard error of the mean). Statistical analysis was performed by using one-way analysis of variance (ANOVA) to compare tumor volumes between groups, using SPSS Base 20.0 software (SPSS Inc, Chicago, IL, USA). Differences were statistically analyzed using multiple comparisons between groups. A log transformation was applied to data to better satisfy the assumptions underlying the analysis. The means and standard errors were computed from untransformed data, but analysis of statistical significance $(\mathrm{p}<0.05)$ was based on transformed data. When necessary, comparison of the means was Bonferroniadjusted.

\section{Results}

Growth inhibition in HeLa cells after chemo-radiotherapy The dose and the schedule of each drug used in the combination assays were selected in accordance with dose-response curves constructed in a previous pilot study. These curves showed that Cisplatin at $0.33 \mu \mathrm{M}$ and 10 Gy of radiation did not show any inhibitory effect on the growth of HeLa cells when administered individually. However, it was observed a significant decrease (approximately $15 \%$ ) with ICI or MF at $10 \mu \mathrm{M}$ (Figure 1).

Cell survival, evaluated by clonogenic assay, is shown for irradiation alone, irradiation with MF or ICI, cisplatin with MF or ICI, cisplatin with irradiation, and cisplatin with irradiation and MF or ICI (Figure 2). Irradiation alone had not effect on the cell proliferation. The antiproliferative effect was comparable when irradiation was applied in combination with cisplatin, or when either irradiation or cisplatin were combined with MF or ICI (about $30-50 \%$ of cell survival compared to the control) (Figure 2A, B). However, when the cells were exposed to a combination of irradiation, cisplatin and MF or ICI, the proliferation was almost completely inhibited. These experiments demonstrate that both agents can act as a chemo-radiosensitizer in cervical cancer cells.

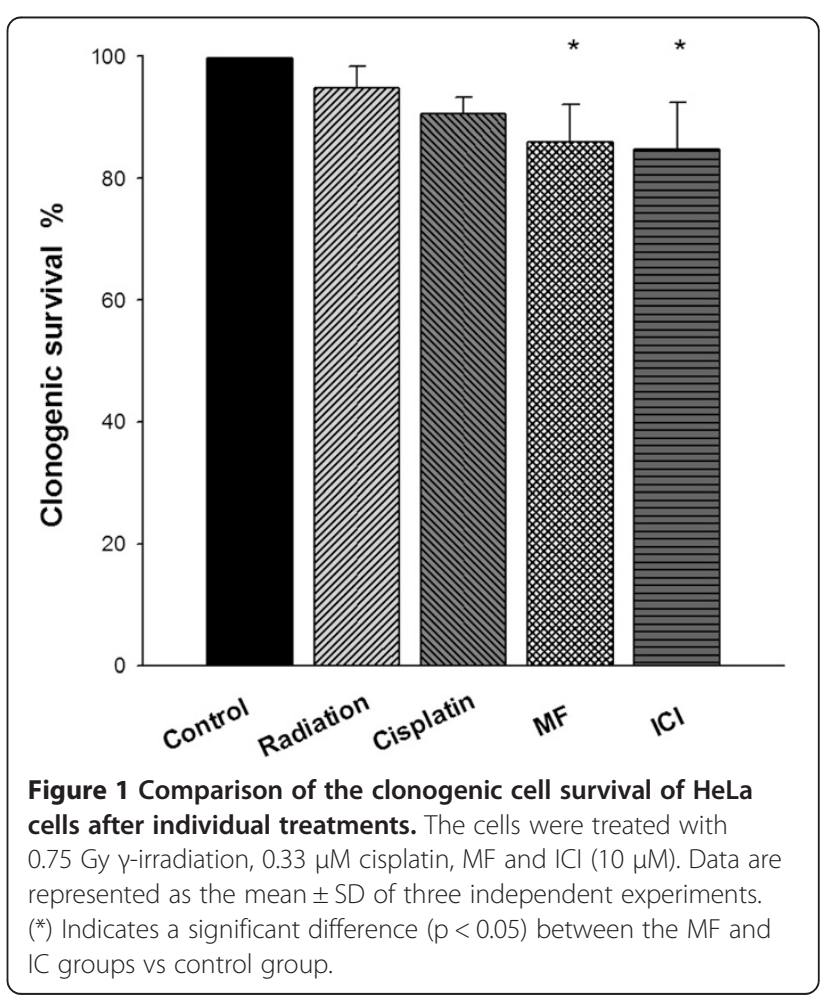




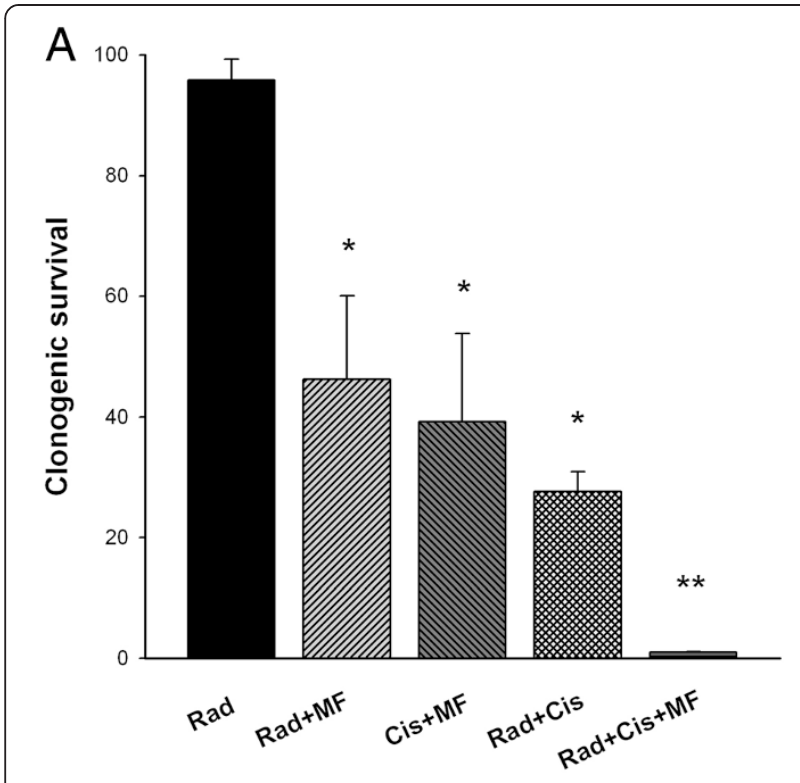

$\mathrm{B}$

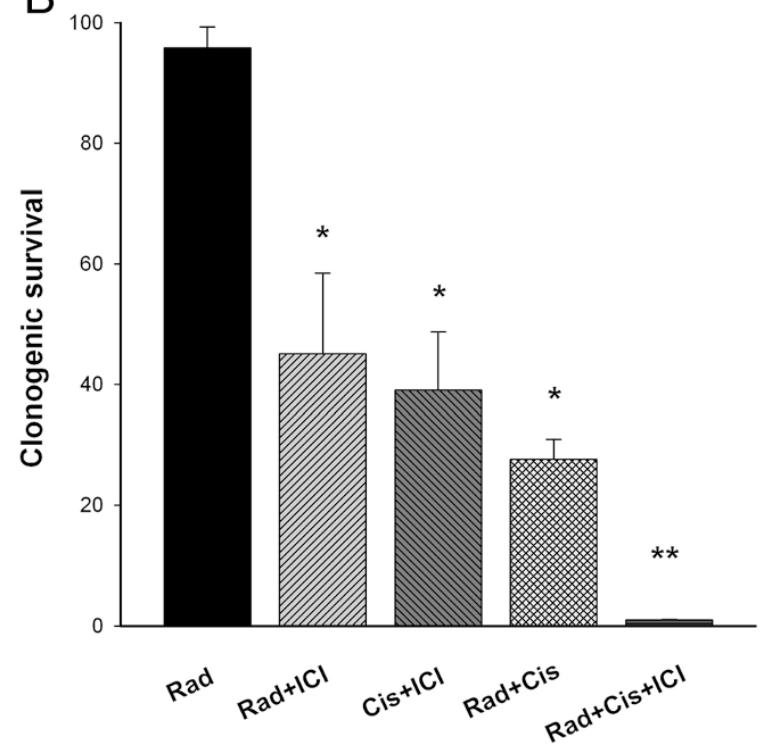

Figure 2 Clonogenic cell survival on HeLa cells after combined treatments. A) Combinations with Mifepristone. B) Combinations with $\mathrm{ICl}$. All clonogenic assays were repeated in duplicate in at least three independent experiments. Values represent the mean \pm SD

\section{Cell cycle and apoptosis analysis}

To determine whether the effects of the combined treatments, using cisplatin and irradiation with MF or ICI on the proliferation of HeLa cells are mediated by inhibition of cell cycle progression, the cell cycle phases of treated cells were analyzed by flow cytometry at 24,48 and $72 \mathrm{~h}$. The results show that the G2/M phase was greater for the treatment with iradiation/cisplatin/ICI than for the treatment with only irradiation/cisplatin: $59.4 \%$ versus
$26.4 \%$ at $24 \mathrm{~h}$ (Figure $3 \mathrm{~A} ; \mathrm{p}<0.001$ ), $27 \%$ versus $22 \%$ at $48 \mathrm{~h}$ (Figure $3 \mathrm{~B}$ ), and $34 \%$ versus $28 \%$ at $72 \mathrm{~h}$ (Figure 3C).

To assess whether growth inhibition by irradiation/cisplatin/ICI was mediated by apoptosis, HeLa cells were stained with Annexin V-PE and 7-ADD for flow cytometric analysis. Externalization of phosphatidylserine to the outer surface of the plasma membrane is a distinct phenomenon of early apoptosis. Analysis of Annexin $\mathrm{V}+1$ 7-AAD- cells (early and late apoptosis) showed that the radiation/cisplatin/ICI treatment increased apoptosis at all times measured with respect to control cells. This change was particularly notable at $72 \mathrm{~h}$, at which time the treatment with radiation/cisplatin/ICI increased apoptosis by $23.8 \%$, whereas treatment with only radiation/cisplatin showed an increase of $2.36 \%$ increase compared to the control (Figure 3D; $\mathrm{p}=0.020$ ). Therefore, the addition of ICI arrested HeLa cells in G2/M phase at 24 and $48 \mathrm{~h}$, and later (at $72 \mathrm{~h}$ ) and induced apoptosis. These data suggest that $\mathrm{G} 2 / \mathrm{M}$ arrested cells underwent apoptosis subsequently after treatment with radiation/cisplatin/ICI. On the other hand, cells exposed to radiation/cisplatin/MF did not show a significant change at any cell cycle phase or in regard to the induction of apoptosis (Figures 3A-D).

\section{Chemo-radiotherapy in tumor xenografts}

Figure 4 shows tumor growth differences for the different treatment combinations: individual treatment (cisplatin, radiation or one of the antihormonals); dual treatment (radiation or cisplatin with one of the antihormonals); triple treatment (radiation and cisplatin with one of the antihormonals). Compared to the triple combination of radiation/cisplatin/MF, there was a statistical difference with the control group from the fourth week on $(\mathrm{p}<0.01)$, with the dual treatments from the eighth week on $(\mathrm{p}<0.05)$, and with the individual treatments from the sixth week on (Figure 4A; $\mathrm{p}<0.01$ ). Whereas compared to the triple combination of radiation/cisplatin/ICI, there was a statistical difference with the control group from the fourth week on $(\mathrm{p}<0.01)$, with the dual treatments from the sixth week on $(\mathrm{p}<0.05)$, and with the individual treatments from the fourth week on (Figure 4B; $\mathrm{p}<0.05$ ).

After ten weeks, it was observed that in the radiation/ cisplatin/MF and radiation/cisplatin/ICI groups, the tumor volume was not different from its initial volume (Figure 5). Contrarily, in the control group the tumor volume was 10 -fold greater than the initial volume and in the radiation/cisplatin group it was 5 -fold greater.

Regarding the toxicity of treatments (Figure 6) no significant change in weight was observed, although there was a tendency to weight loss with the dual and triple treatment groups. In the latter cases, the weight of the animals was recovered by the end of the study. 


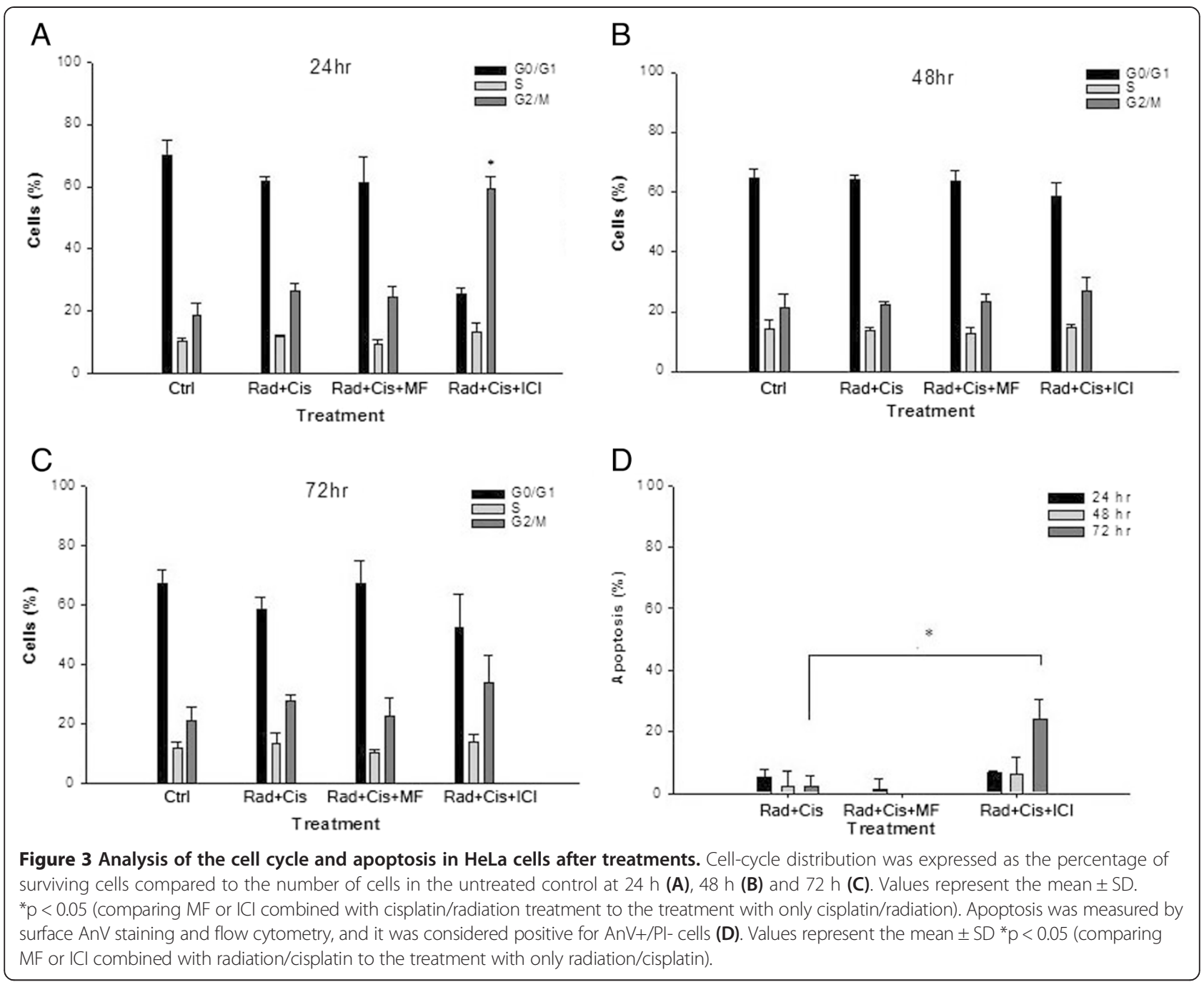

\section{VEGF relative expression}

The effect of the triple treatments using cisplatin with one of the antihormonal compound on the VEGF mRNA levels was analysed in tumors at the end of the study. The triple combination of radiation/cisplatin/MF and radiation/cisplatin/ICI showed a statistically significant lower level of VEGF than that found in the control and radiation/cisplatin groups (Figure 7). Treatment with cisplatin and irradiation did not modify VEGF mRNA levels in tumors with respect to those from control group.

\section{Discussion}

Currently, treatment for advanced cervical cancer involves the combination of cisplatin-based chemotherapy and radiotherapy. However, this treatment generates resistance and toxicity, either by the side effects caused by cisplatin (neurotoxicity, myelotoxicity, nephrotoxicity and ototoxicity) or the side effects caused by radiotherapy (damage to the normal tissue). Although this combination (cisplatin/radiation) has proved to be effective in the early stages of the disease, resulting in a patient survival rate of around $90 \%$, its use for patients in advanced stages has led to a survival rate of only about $17 \%[7,8]$. The poor response to treatment is mainly caused by chemoradioresistant cells, which are capable of provoking uncontrolled local tumor growth.

Different efforts have been done to improve chemoradiotherapy for cervical cancer. However, there are few studies have attempted to identify potential agents as chemoradiosensitizer. Several compounds no-related to anti-hormonals agents have showed a potential radiosensitizer effect. Aspirin has showed radiosensitizing effect on human cervical cancer cells, its mechanism was mediated by Bcl-2, caspasas-3 pathway and p53 [24]. Plumbagin (naturally occurring naphtquinone) has been studied as radiosensitizer on HeLa, SiHa and C33A cells; results showed that this compound in combination with 


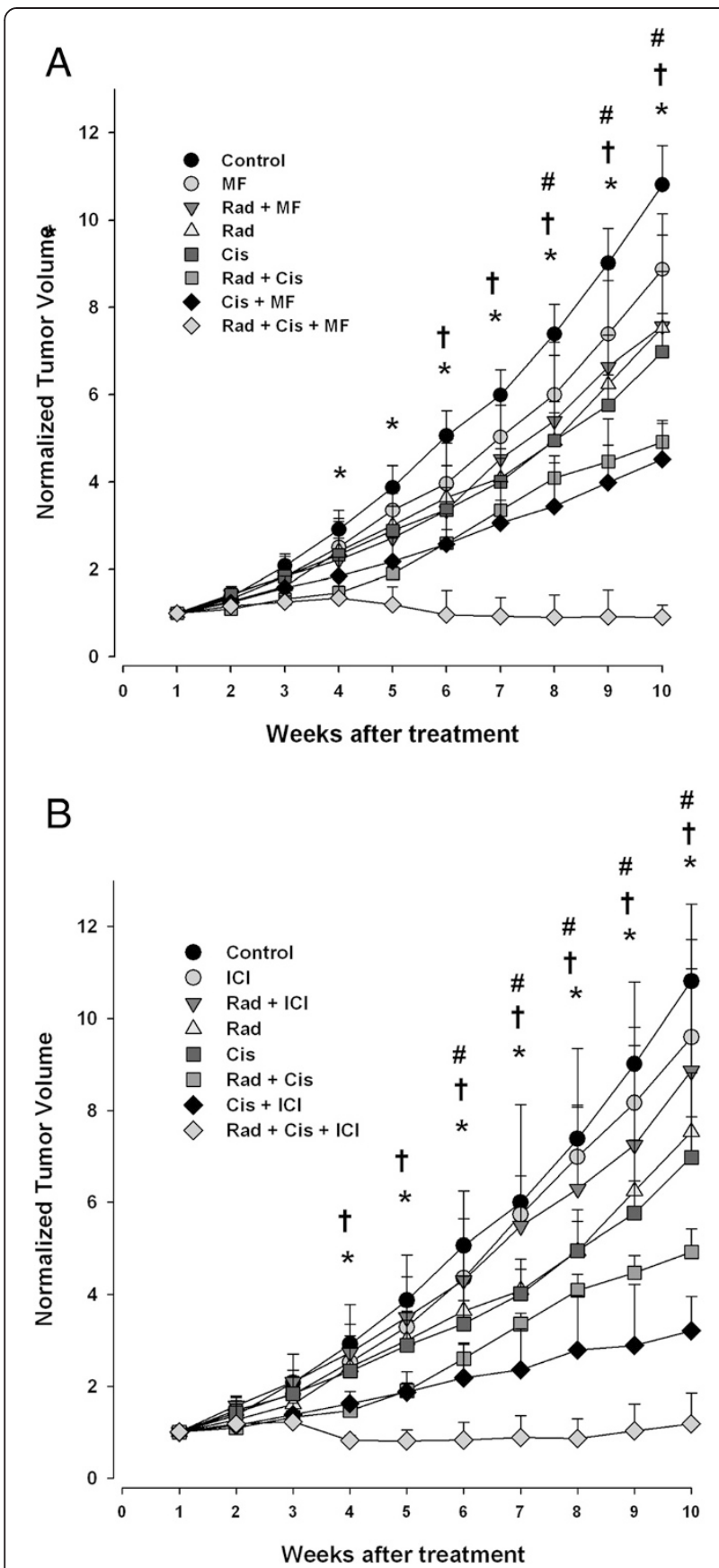

Figure 4 Efficacy of antihormonal agents on HeLa cell tumors treated with cisplatin or radiotherapy. HeLa cells were implanted s.c. in both hind limbs of nude mice. Treatment was initiated when the tumors reached $150 \mathrm{~mm}^{3}$. Mifepristone (A) or ICl (B) were administered in combination with radiation, cisplatin, and radiation/cisplatin. B) ICl was also administered in combination with radiation, cisplatin, and radiation/cisplatin. As controls, tumor growth was determined for mice treated only with the vehicle. Data are presented as the mean \pm SEM of five to six animals. $\left(^{*}\right)$ indicates a significant difference $(p<0.01)$ between the triple combination groups vs control group; (\#) and ( + ) represent a significant difference vs the dual treatments $(p<0.05)$ and individual treatments $(p<0.01)$ respectively.

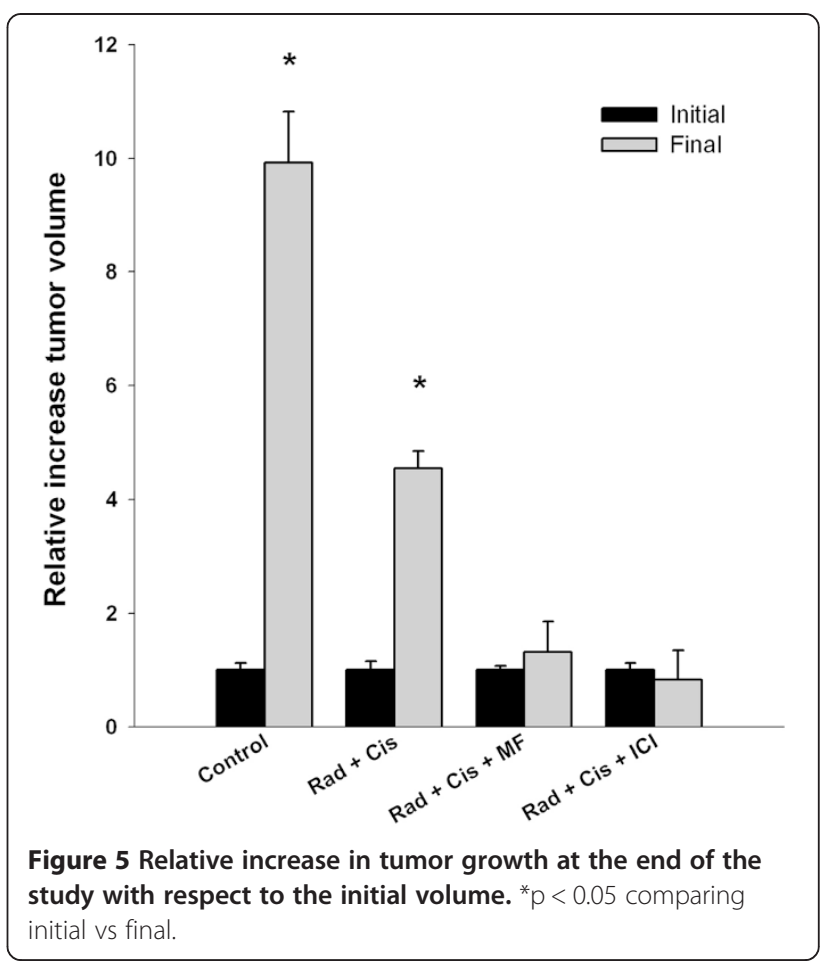

radiation augmented cell growth inhibition through of the apoptosis pathway [25]. Tillmans et al. [26] evaluated the radiosensitization of cervical cancer cell lines by retinoic acid demonstrating that the effect involves the HPV and p53 status. Furthermore, some drugs used in

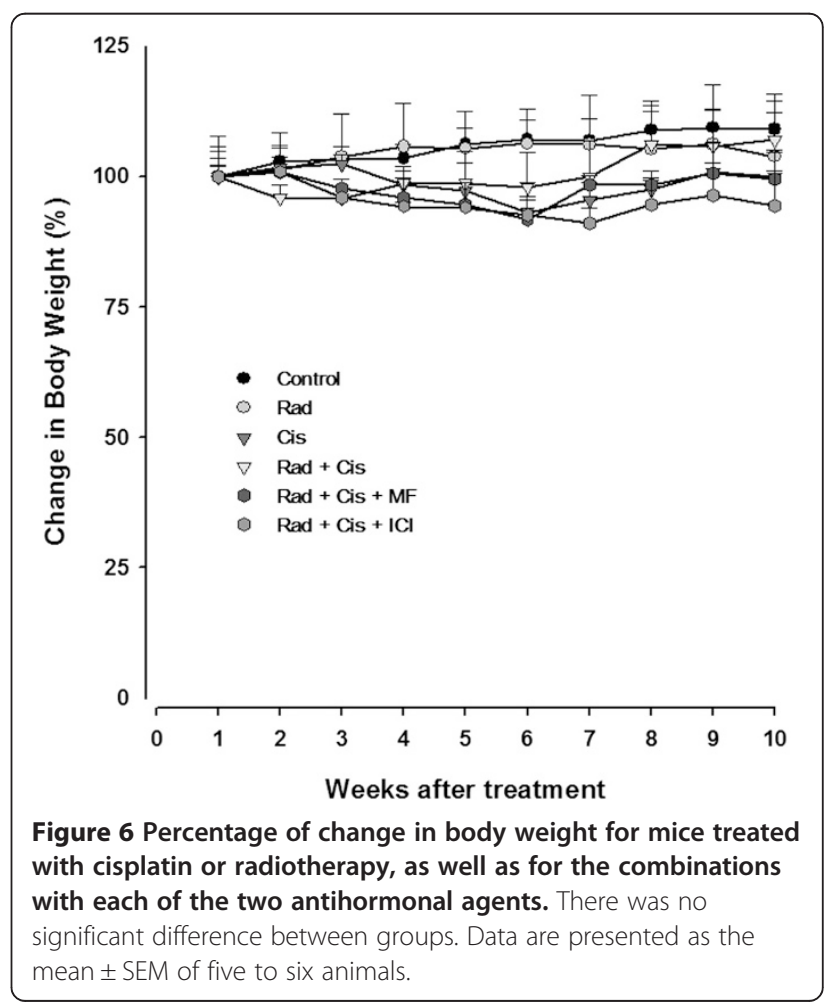




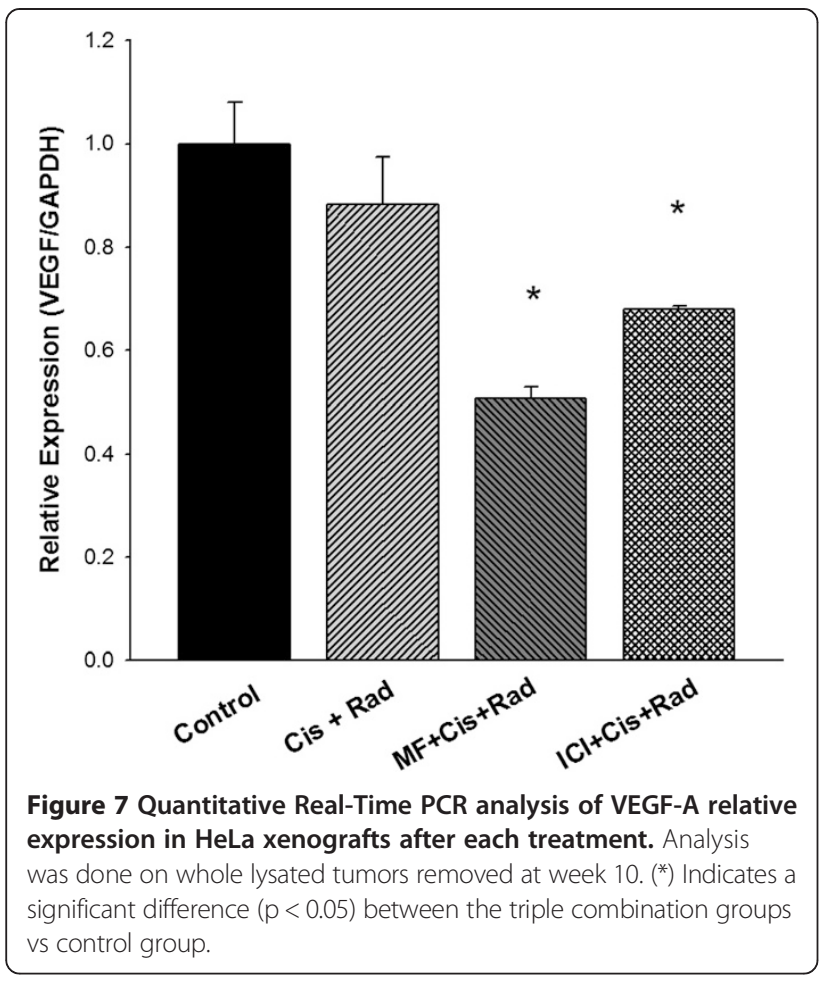

the chemotherapy as hydroxyurea, gemcitabine, taxanes have showed a radiosensitizing effect; however greater side effects can be found with these drugs.

In some types of hormone-dependent cancers, such as breast and ovarian, anti- hormonal agents have been used as chemo-sensitizer agents. However the application of these compounds as chemo-radio-sensitizers has been poorly studied in cervical cancer. Hence, the purpose of the present study was to investigate whether MF (an antiprogestin) or ICI (an antiestrogen) used simultaneously with cisplatin and radiotherapy could produce a synergism and increase the anti-proliferative effect in a cervical cancer cell line (HeLa) and in xenostransplants treated with cisplatin and radiation.

Prior to evaluating the effect of each agent, cisplatin and radiation doses were established for the combined assays. The concentration of drugs applied individually did not have a significant cytotoxic effect, thus a possible synergistic effect would only be found when combinations are applied. The individual dose of radiation and cisplatin used in the present study showed no more than $25 \%$ of cell death. The concentration of the antihormonals $(10 \mu \mathrm{M})$, employed herein had no significant effect when these agents were applied individually. When this concentration, which is close to plasma concentrations achievable in humans, was used in previous studies, the anti- hormonal agent showed a chemo-sensitizer effect for cisplatin in ovarian [13] and cervical carcinoma cell lines [19,21], and for doxorubicin in hepatoma and leukemia cell lines [27]. This same concentration had a chemosensitizing effect with doxorubicin in breastcancer cell lines [28] and a growth inhibition effect in ovarian cell lines [29].

Results of the clonogenic assay showed that MF has a chemo-radiosensitizer effect on the HeLa cell line. Cell survival decreased nearly $100 \%$ when MF was added to the standard treatment of cisplatin with radiation. It has been reported that MF inhibits proliferation of certain types of hormone-dependent cancers, such as breast cancer positive for the progesterone receptor [30,31], ovarian cancer [32,33], endometrium cancer [34], prostate cancer [35] and gastric cancer [36]. Cervical cancer does not respond to hormonal treatment. However, a study conducted in our laboratory demonstrated that when combined with MF, there was a significant increase in the cytotoxicity of cisplatin in two cervical cancer cell lines, HeLa and CaSki [19]. Tieszen CR et al. [37] reported that growth inhibition of cancer cells by antiprogestin MF is not dependent upon the expression of nuclear progesterone receptors. They showed that MF is capable of inhibiting the growth of in vitro cancer cells derived from the nervous system, breast, prostate, ovary and bone. Nearly all of these cancer cells lack the expression of classic nuclear progesterone receptors.

As a chemosensitizing agent, MF has been used to modulate the cytotoxic activity of doxorubicin, paclitaxel and cisplatin, mostly in hormone-dependent cancers such as breast cancer [16] and ovarian cancer [17,18]. However, there are few studies that demonstrate the role of MF as chemo-radiosensitizer agent. We previously reported that MF could improve the efficacy of chemoradiotherapy in glioblastoma xenografts [20].

The addition of MF to the standard treatment of cisplatin with radiation did not induce significant changes in the cell cycle distribution. Previous studies have shown that MF induces G1-S blockage of the cell cycle through inhibition of cdk2 activity in human ovarian cancer cells [38]. A reduction in cdK2 activity has been associated with the inhibition of the transcription factor E2F1, which modulates S-phase progression [39]. The present study showed no changes in the G1 phase of the cell cycle when MF was added to chemoradiotherapy treatment. Nevertheless, the dose of MF used in our study was lower than that used in previous reports. Furthermore, HeLa cell line shows low expression of hormonal receptors $[21,40]$.

Many studies have reported the apoptotic effect of MF on different tumor types [41-47]. Recently it was reported that MF at low concentrations $(<10 \mu \mathrm{M})$ could enhance the chemosensitivity of cancer cells to cisplatin, thus increasing the capability of this compound to induce apoptosis in HeLa cells. The greater effect of cisplatin on growth inhibition induced by MF was associated with the 
down-regulation of the HPV E6, survivin protein and the upregulation of the p53 protein [48]. Nevertheless, in the present study there were no significant changes in apoptosis when MF was added.

Whereas cell survival was reduced with the addition of MF to the cisplatin/radiation treatment (according to the clonogenic assay), no significant change was found in apoptosis or the cell cycle. The clonogenic assay involves a longer exposure (15 days) to treatment than that used in the analysis of apoptosis or the cell cycle (24 to $72 \mathrm{~h}$ ). This could be one of the causes of the observed difference. On the other hand, when in the combined MF/cisplatin/radiation treatment involved a higher dose of cisplatin $(3.3 \mu \mathrm{M})$, there was an increase in the percentage of cells in the G2/M phase at $72 \mathrm{~h}$ (data not shown). Therefore, exposure to MF for longer times or the use of higher concentrations of cisplatin could sensitize HeLa cell line by inducing G2/M arrest, the most radiosensitive cell cycle phase $[49,50]$.

The results of the present study demonstrate that ICI also has a chemo-radiosensitizing effect on the HeLa cell line. The results show a decrease in survival with cells exposed to the combined treatment of ICI/cisplatin/radiation. The cytotoxic effect of the antiestrogen ICI in combination with certain antineoplastic agents has been clearly demonstrated in hormone-dependent breast cancer, reducing cellular proliferation and increasing cytotoxicity [51]. There is also evidence that the combination of cisplatin with ICI produces a synergistic effect, increasing cellular cytotoxicity in a negative estrogen receptor ovarian cancer line (A2780) [13]. A study conducted in our laboratory demonstrated that the combination of cisplatin with ICI produces a synergistic antiproliferative effect in cervical cancer cell lines. The effect of ICI on the cytotoxicity of cisplatin could be mediated, at least in part, by cell cycle arrest at the G2/M phase [21]. This correlates with the results obtained in the present study, where analysis of the cell cycle at 24 hours showed an increase in the percentage of cells arrested at G2/M when the ICI/cisplatin/ radiation treatment was administered. Furthermore, there was an induction of apoptosis at $72 \mathrm{~h}$. Therefore, the pathway of ICI to sensitize HeLa cell line to chemoradiotherapy, is $\mathrm{G} 2 / \mathrm{M}$ arrest preceding apoptosis, which may be a mechanism for its inhibitory effects on growth of the cancer cells. With the MF/cisplatin/radiation treatment, we hypothesize that the cisplatin dose should have been greater in order to arrest the cell cycle at the G2/M phase and induce apoptosis. The activity of MF appears to depend on the time of exposure to the treatment or the concentration of cisplatin used.

The response biological to progesterone is mediated by two isoforms of the progesterone receptor, PR-A and PR-B. In most cell lines such as MCF-7 (breast cancer cells), CV-1 (monkey kidney fibroblast), and HeLa (cervical carcinoma cells), PR-A functions as a transcriptional repressor, whereas PR-B functions as a transcriptional activator of progesterone-responsive genes [52]. Furthermore, it was reported that PR-A but not PR-B, in the presence of either progesterone or anti-progestin, inhibited ER-mediated transcriptional activity [53]. The same authors have reported that mifepristone was capable of functioning as an antagonist of ER only in the presence of PR-A in MCF-7 cells [52]. We previously reported that $\mathrm{ER} \alpha$ and $\mathrm{PR}-\mathrm{AB}$ gene levels in HeLa cells were relatively low compared to those observed in MCF-7 cells [21]; however, ICI was able to downregulated ER and PR genes, demonstrating a light ligand-receptor interaction. It is possible that MF could show similar results and its effect chemo-sensitization can be explained partially by this mechanism. Further studies will need to be performed to confirm this hypothesis.

In the present study we also evaluated whether there is an improvement in the response of xenotransplants of cervix in vivo with the addition of MF or ICI to the standard therapy of cisplatin and radiotherapy. We observed significant differences in tumor volume between the treatments. When the MF or ICI were combined with cisplatin, the effect was comparable to that of the standard treatment (cisplatin/radiation). However, there was a notable reduction in the tumor growth- rate when MF or ICI was added to the cisplatin/radiation scheme, suggesting that MF and ICI play an important role in the chemo-radiosensitization not only in-vitro but also in- vivo. When we evaluated the VEGF expression in the xenografts at the end of the study, we observed that both antihormonal agents decrease VEGF production. However, the effect was more evident with MF, suggesting that VEGF down-regulation is one of the mechanisms by this compound acts in combination with cisplatin/ radiation.

Other mechanisms independent of ER/PR that could be involved in the chemoradiosensitizing effect of MF and ICI are the decreasing the insulin-like growth factor receptor (IGF-1), decreasing transforming-growth factor$\beta 1$ (TGF- $\beta 1$ ) or loss c-fos expression.

On the other hand, it has reported that some tumour suppressor genes are involved in the regulation of angiogenesis. One of them, p53, has been shown to be associated with VEGF in non-small cell lung cancer [54]. The induction of VEGF gene expression by hypoxia in tumor cells involves both an increase in the rate of gene transcription mediated by HIF-1 (hypoxia-inducible factor), and an enhancement of the stability of VEGF mRNA. Transcription of VEGF mRNA is also induced by a variety of growth factors and cytokines, including PDGF (platelet-derived growth factor), EGF (Epidermal growth factor), TNF $\alpha$ (tumor necrosis factor), TGF- $\beta 1$ (Tumoral growth factor), and IL-1 $\beta$ (interleukin) [55]. Also other 
genes as $\mathrm{Bcl} 2$ have been associated with neo-angiogenesis and a worse prognosis [56]. Moreover the loss of intracrine VEGF signaling leads to an increase in spontaneous apoptosis and chemosensitivity. These effects were mediated via upregulation of the proapoptotic mediators as caspase-3, and Bax [56].

Recent studies have showed that tumor cells express TLRs (Toll-like receptors), and this expression can facilitate the tumor development [57]. However, the relationship between TLR-8 and cervical cancer has been little studied. Recently, it has been reported the involvement of TLR-8 and its relationship with VEGF in cervical cancer [58]. The authors demonstrated increased expression of TLR-8 in HeLa cells and in cervical cancer tissue from patients; in this study also was evaluated the correlation between TLR- 8 expression and two genes associated to the pathogenesis of cancer like Bcl-2 and VEGF. They found a positive correlation between TLR-8 and $\mathrm{Bcl} 2$ or VEGF expression both in cervical cancer tissues as well as HeLa cells. These results suggest that TLR- 8 may be a therapeutic target in cervical cancer and its ligand can modulate the response of chemotherapy or radiotherapy. According to our results, it would be interesting to see in future studies, if the pathway TLR-8 is involved in the low expression of VEGF by the triple combinations of radiation/cisplatin/MF and radiation/cisplatin/ICI.

Finally, the lack of significant change in body weight of animals, suggest that both Mifepristone and ICI could be safely administered at these doses.

\section{Conclusions}

The present study shows that the addition of Mifepristone or ICI improves chemo-radiotherapy treatment. It is possible that either of these antihormonal treatments sensitizes to HeLa cell line by inducing G2/M arrest, the most radiosensitive cell cycle phase, and by enhancing the capacity of cisplatin to induce apoptosis. Hence, the results strongly suggest that either antihormonal agent, when used in combination with cisplatin and radiation, could have potential as a chemo-radio-sensitizer for cervical cancer treatment.

\section{Abbreviations}

HPV: Human papillomavirus; MF: Mifepristone; ICl: ICI 182,780;

DMEM: Dulbeccós modified Eagle's medium;

EDTA: Ethylenediaminetetracetic acid; PE: Plating efficiency;

s.c: Subcutaneously; VEGF: Vascular endothelial growth factor;

RT-PCR: Reverse-transcriptase polymerase chain reaction; TC: Threshold cycle; cdk2: Cyclin-dependent kinase 2.
}

\section{Competing interests}

The authors declare that they have no competing interests.

\section{Authors' contributions}

PGL planned the studies, coordinated all activities, performed analysis of results, and was involved in the experimental procedures. MSM and LAM participated in the experimental procedures of chemo-radiotherapy and data processing. RJ participated in the tumor model design, chemotherapy procedures and VEGF expression experiments. RM and HPG participated in cell cycle and apoptosis experiments and analysis. All authors read and approved the final manuscript.

\section{Acknowledgements}

This work was partially funded by CONACyT-Mexico grant 44386. The authors would like to thank M.Sc. Miguel Rodriguez-Ponce, Oscar Angeles-Zaragoza and the personnel from the Radiotherapy Department at INCan for their help during the irradiation procedures. We also thank to Allan Larsen who provided medical revision of the manuscript.

\section{Author details}

${ }^{1}$ Instituto Nacional de Cancerología, Subdirección de Investigación Básica, México D.F. 14080, México. ${ }^{2}$ Posgrado en Ciencias Biológicas, Universidad Nacional Autónoma de México, México D.F. 04510, México. ${ }^{3}$ Instituto de Física, Universidad Nacional Autónoma de México, México D.F. 04510, México. ${ }^{4}$ Unidad de Investigación Biomédica en Cáncer INCan-UNAM, Instituto Nacional de Cancerología, México D.F. 14080, México. ${ }^{5}$ Instituto Nacional de Enfermedades Respiratorias, Departamento de Enfermedades Crónico-Degenerativas, México D.F, Mexico.

Received: 31 August 2014 Accepted: 8 January 2015

Published online: 27 January 2015

\section{References}

1. Bosch FX. Epidemiology of human papillomavirus infections: new options for cervical cancer prevention. Salud Publica Mex. 2003;45:S326-39.

2. Lörincz AT. Screening for cervical cancer: new alternatives and research. Salud Publica Mex. 2003:45:S376-87.

3. Walboomers JM, Jacobs MV, Manos MM, Bosch FX, Kummer JA, Shah KV, et al. Human papillomavirus is a necessary cause of invasive cervical cancer worldwide. J Pathol. 1999;189(1):12-9.

4. Rose PG. Chemoradiotherapy for cervical cancer. Eur J Cancer. 2002;38:270-8.

5. Takashi N, Tatsuya O, Hitoshi I, Yoshiyuki S, Takeo T. Current advancement in radiation therapy for uterine cervical cancer. J Radiat Res. 2010;51:1-8.

6. Eifel PJ, Winter K, Morris M, Levenback C, Grigsby PW, Cooper J, et al. Pelvic irradiation with concurrent chemotherapy versus pelvic and para-aortic irradiation for high-risk cervical cancer. J Clin Oncol. 2004;22:872-80.

7. Morris M, Eifel PJ, Lu J, Grigsby PW, Levenback C, Stevens RE, et al. Pelvic radiation with concurrent chemotherapy compared with pelvic and para-aortic radiation for high-risk cervical cancer. N Engl J Med. 1999;340:1137-43.

8. Rose PG, Bundy BN, Watkins EB, Thigpen JT, Deppe G, Maiman MA, et al. Concurrent cisplatin-based radiotherapy and chemotherapy for locally advanced cervical cancer. N Engl J Med. 1999;340:1144-53.

9. Benedetti PP, Greggi S, Scambia G. High-dose cisplatin and bleomycin neoadjuvant chemotherapy plus radical surgery in locally advanced cervical carcinoma: a preliminary report. Gynecol Oncol. 1991;41:212.

10. Soutter WP, Leake RA. Steroid Hormone Receptors in Gynaecological Cancer. In: Bonnard J, editor. Recent Advances in Obstetrics and Gynaecology. Edinburgh: Churchill Livingstone; 1987. p. 175.

11. Winston R, Kao PC, Kiang DT. Regulation of insulin-like growth factors by antiestrogen. Breast Cancer Res Treat. 1994;31(1):107-15.

12. Jain JK, Li A, Yang W, Minoo P, Felix JC. Effects of mifepristone on proliferation and apoptosis of human endometrium in new users of medroxyprogesterone acetate. Hum Reprod. 2006;21(3):798-809.

13. Ercoli A, Battaglia A, Raspaglio G, Fattorossi A, Alimonti A, Petrucci F, et al. Activity of cisplatin and ICI 182,780 on estrogen receptor negative ovarian cancer cells: cell cycle and cell replication rate perturbation, chromatin texture alteration and apoptosis induction. Int J Cancer. 2000;85(1):98-103.

14. Kamradt MC, Mohideen N, Vaughan AT. RU486 increases radiosensitivity and restores apoptosis through modulation of HPV E6/E7 in dexamethasonetreated cervical carcinoma cells. Gynecol Oncol. 2000;77(1):177-82.

15. Riggins RB, Bouton AH, Liu MC, Clarke R. Antiestrogens, aromatase inhibitors, and apoptosis in breast cancer. Vitam Horm. 2005;71:201-37.

16. Huang J, Zhang $Y$, Huang $Y$, Zhang $X$, Xiao J. Reversal effect of mifepristone on adriamycin resistance in human breast cancer cell line MCF-7/ADM in vitro and in vivo. Zhong Nan Da Xue Xue Bao Yi Xue Ban. 2010;35:576-83.

17. Gamarra-Luques CD, Goyeneche AA, Hapon MB, Telleria CM. Mifepristone prevents repopulation of ovarian cancer cells escaping cisplatin-paclitaxel therapy. BMC Cancer. 2012;12:200. 
18. Freeburg EM, Goyeneche AA, Telleria CM. Mifepristone abrogates repopulation of ovarian cancer cells in between courses of cisplatin treatment. Int J Oncol. 2009;34:743-55.

19. Jurado R, Lopez-Flores A, Alvarez A, Garcia-Lopez P. Cisplatin cytotoxicity is increased by mifepristone in cervical carcinoma: an in vitro and in vivo study. Oncol Rep. 2009;22:1237-45.

20. Llaguno-Munive M, Medina LA, Jurado R, Romero-Pina M, Garcia-Lopez P. Mifepristone improves chemo-radiation response in glioblastoma xenografts. Cancer Cell Int. 2013;13:29.

21. García-López P, Rodríguez-Dorantes M, Enrique Perez-Cardenas E, Cerbon M, Mohar-Betancourt A. Synergistic effects of ICI 182,780 on the cytotoxicity of cisplatin in cervical carcinoma cell lines. Cancer Chemother Pharmacol. 2004:53:533-40.

22. Munshi A, Hobbs M, Meyn RE. Clonogenic Cell survival Assay. In: Blumenthal $\mathrm{RD}$, editor. Methods in Molecular Medicine. Volume 110: Chemosensitivity. 1st ed. Humana Press Inc: Totowa, New Jersey; 2005. p. 21-8.

23. Medina LA, Herrera-Penilla BI, Castro-Morales MA, García-López P, Jurado R, Pérez-

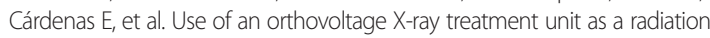
research system in a small-animal cancer model. J Exp Clin Cancer Res. 2008;27:57.

24. Kim KY, Seo JY, Jeon GA, Nam MJ. The combined treatment of aspirin and radiation induces apoptosis by the regulation of bcl-2 and caspase- 3 in human cervical cancer cell. Cancer Lett. 2003;189(2):157-66.

25. Nair S, Nair RR, Srinivas P, Srinivas G, Pillai MR. Radiosensitizing effects of plumbagin in cervical cancer cells is through modulation of apoptotic pathway. Mol Carcinog. 2008;47(1):22-33.

26. Tillmanns TD, Kamelle SA, Guruswamy S, Gould NS, Rutledge TL, Benbrook DM. Sensitization of cervical cancer cell lines to low-dose radiation by retinoic acid does not require functional p53. Gynecol Oncol. 2005;97(1):142-50

27. Lecureur V, Fardel O, Guillouzo A. The antiprogestatin drug RU 486 potentiates doxorubicin cytotoxicity in multidrug resistant cells through inhibition of P-glycoprotein function. FEBS Lett. 1994;355:187-91.

28. De Vicenzo R, Scambia G, Benedetti P, Fattorossi A, Bonanno G, Ferlini C, et al. Modulatory effect of tamoxifen and $\mathrm{ICI} 182,780$ on adriamycin resistance in MCF-7 human breast-cancer cells. Int J Cancer. 1996:4(68(3)):340-8.

29. Goyeneche AA, Seidel EE, Telleria CM. Growth inhibition induced by antiprogestins RU-38486, ORG-31710, and CDB-2914 in ovarian cancer cells involves inhibition of cyclin dependent kinase 2. Invest New Drugs. 2012;30(3):967-80.

30. Liang Y, Hou M, Kallab AM, Barrett JT, El Etreby F, Schoenlein PV. Induction of antiproliferation and apoptosis in estrogen receptor negative MDA-231 human breast cancer cells by mifepristone and 4-hydroxytamoxifen combination therapy: a role for TGFbeta1. Int J Oncol. 2003;23:369-80.

31. El Etreby MF, Liang Y, Wrenn RW, Schoenlein PV. Additive effect of mifepristone and tamoxifen on apoptotic pathways in MCF-7 human breast cancer cells. Breast Cancer Res Treat. 1998;51:149-68.

32. Rose FV, Barnea ER. Response of human ovarian carcinoma cell lines to antiprogestin mifepristone. Oncogene. 1996;12(5):999-1003.

33. Rocereto TF, Saul HM, Aikins Jr JA, Paulson J. Phase II study of mifepristone (RU486) in refractory ovarian cancer. Gynecol Oncol. 2000;77:429-32.

34. Navo MA, Smith JA, Gaikwad A, Burke T, Brown J, Ramondetta LM. In vitro evaluation of the growth inhibition and apoptosis effect of mifepristone (RU486) in human Ishikawa and HEC1A endometrial cancer cell lines. Cancer Chemother Pharmacol. 2008;62:483-9.

35. Zhang H, Lu JJ, Gao QZ, Zhang J. Induction of apoptosis by mifepristone in androgen-independent prostate cancer cell lines in vitro. Zhonghua Wai Ke Za Zhi. 2006:44:382-5.

36. Li DQ, Wang ZB, Bai J, Zhao J, Wang Y, Hu K. Effects of mifepristone on proliferation of human gastric adenocarcinoma cell line SGC-7901 in vitro. World J Gastroenterol. 2004;10:2628-31.

37. Tieszen CR, Goyeneche AA, Brandhagen BN, Ortbahn CT, Telleria CM. Antiprogestin mifepristone inhibits the growth of cancer cells of reproductive and non-reproductive origin regardless of progesterone receptor expression. BMC Cancer. 2011;11:207.

38. Goyeneche AA, Carón RW, Telleria CM. Mifepristone inhibits ovarian cancer cell growth in vitro and in vivo. Clin Cancer Res. 2007;13:3370-9.

39. Musgrove EA, Lee CS, Cornish AL, Swarbrick A, Sutherland RL. Antiprogestin inhibition of cell cycle progression in T-47D breast cancer cells is accompanied by induction of the cyclin-dependent kinase inhibitor p21. Mol Endocrinol. 1997;11:54-66

40. Leblanc K, Sexton É, Parent S, Bélanger G, Déry M, Boucher V, et al. Effects of 4-hydroxytamoxifen, raloxifene and ICI 182780 on survival of uterine cancer cell lines in the presence and absence of exogenous estrogens. Int J Oncol. 2007;30(2):477-87.

41. Sun QL, Zhang XG, Xing QT, Ding P, Feng JB, Wu XP, et al. A study of mifepristone/IFN- $\gamma$-induced apoptosis of human cholangiocarcinoma cell line FRH-0201 in vitro. Onco Targets Ther. 2012;5:335-42.

42. Gaddy V, Barret J, Delk J. Mifepristone induced growth arrest caspase activation and apoptosis of estrogen receptor expressing antiestrogen resistant breast cancer cell. Clin Cancer Res. 2004;10:5215-25.

43. Han S, Sidell N. RU 486 induced growth inthibition of human endometrial cells involves the nuclear factor kappa B signaling pathway. J Clin Endocrinol Metab. 2003;88:713-9.

44. Skor MN, Wonder EL, Kocherginsky M, Goyal A, Hall BA, Cai Y, et al. Glucocorticoid receptor antagonism as a novel therapy for triple-negative breast cancer. Clin Cancer Res. 2013;19:6163-72.

45. Fauvet $R$, Dufournet Etienne $C$, Poncelet $C$, Bringuier AF, Feldmann G, Darai E. Effects of progesterone and anti-progestin (mifepristone) treatment on proliferation and apoptosis of the human ovarian cancer cell line, OVCAR-3. Oncol Rep. 2006;15:743-8.

46. Jang JH, Woo SM, Um HJ, Park EJ, Min KJ, Lee TJ, et al. RU486, a glucocorticoid receptor antagonist, induces apoptosis in U937 human lymphoma cells through reduction in mitochondrial membrane potential and activation of p38 MAPK. Oncol Rep. 2013;30:506-12.

47. Fjelldal R, Moe BT, Orbo A, Sager G. MCF-7 cell apoptosis and cell cycle arrest: non-genomic effects of progesterone and mifepristone (RU-486). Anticancer Res. 2010;30:4835-40.

48. Li C, Ye H. Mifepristone sensitizing cisplatin for cervical adenocarcinoma HeLa cell sensitivity to chemotherapy and its mechanism. Eur J Gynaecol Oncol. 2013;34:142-7.

49. Homback N, Shen R, Shidnia H, Kaiser H. Synergistic cytotoxic and antitumor effects of irradiation and taxol on human HeLa cervix carcinoma and mouse B16 melanoma cells. In vivo. 1994;8(5):819-23.

50. Liu C, Chang H, Chen I, Chen C, Hsu M, Ful S, et al. Costunolide causes mitotic arrest and enhances radiosensitivity in human hepatocellular carcinoma cells. Radiat Oncol. 2011;6:2-8.

51. Ikeda H, Taira N, Nogami T, Shien K, Okada M, Shien T, et al. Combination treatment with fulvestrant and various cytotoxic agents (doxorubicin, paclitaxel, docetaxel, vinorelbine, and 5-fluorouracil) has a synergistic effect in estrogen receptor-positive breast cancer. Cancer Sci. 2011;102(11):2038-42.

52. Giangrande PH, Pollio G, McDonnell DP. Mapping and characterization of the functional domains responsible for the differential activity of the A and $B$ isoforms of the human progesterone receptor. J Biol Chem. 1997:272(52):32889-900.

53. McDonnell DP, Goldman ME. RU486 exerts antiestrogenic activities through a novel progesterone receptor A form-mediated mechanism. J Biol Chem. 1994;269(16):11945-9.

54. Fontanini $\mathrm{G}$, Boldrini L, Vignati S, Chinè S, Basolo F, Silvestri $V_{\text {, et }}$ al. $\mathrm{BCl} 2$ and p53 regulate vascular endothelial growth factor (VEGF)-mediated angiogenesis in non-small cell lung carcinoma. Eur J Cancer. 1998:34(5):718-23.

55. McMahon G. VEGF receptor signaling in tumor angiogenesis. Oncologist. 2000:5(1):3-10.

56. Samuel S, Fan F, Dang LH, Xia L, Gaur P, Ellis LM. Intracrine vascular endothelial growth factor signaling in survival and chemoresistance of human colorectal cancer cells. Oncogene. 2010;30(10):1205-12.

57. Basith S, Manavalan B, Yoo TH, Kim SG, Choi S. Roles of toll-like receptors in cancer: a double-edged sword for defense and offense. Arch Pharm Res. 2012;35(8):1297-31.

58. Zhang $Y$, Yang $H$, Barnie PA, Yang $P, S u Z$, Chen J, et al. The expression of Toll-like receptor 8 and its relationship with VEGF and BCl-2 in cervical cancer. Int J Med Sci. 2014;11(6):608-13. 